\title{
Evaluating the effects of vanadyl sulfate on biomarkers of oxidative stress and inflammation in renal tissue of rats with diabetes type 2
}

\author{
Bahmani Fereshteh ${ }^{1}$, Afsharipour Ali-Reza1, Manteghie Nastaran', \\ Taghizadeh Mohsen', Mirhashemi Seyyed Mehdi ${ }^{\circledR 2 *}$ \\ ${ }^{1}$ Research Center for Biochemistry and Nutrition in Metabolic Diseases, Kashan University of \\ Medical Sciences, Kashan, Iran, ${ }^{2}$ Metabolic Diseases Research Center, Research Institute for \\ Prevention of Non-Communicable Diseases, Qazvin University of Medical Sciences, Qazvin, Iran.
}

\begin{abstract}
Vanadyl sulfate (VS) is an ingredient in some food supplements and experimental drugs. This study was designed to assay the effects of VS on biomarkers of oxidative stress and inflammation in renal tissue of rats with diabetes type 2.30 male Wistar rats were divided into three equal groups as follow: non-diabetics, non-treated diabetics and VS-treated diabetics. Diabetes type 2 has been induced through high fat diet and fructose in the animals. Diabetic rats were treated with $25 \mathrm{mg} / \mathrm{kgBW}$ of VS in water for 12 weeks. At the end of study, glucose and insulin were measured using commercially available kits in serum and biomarkers of oxidative stress and inflammation in renal homogenates of animals were measured by related methods. Compared to controls, glucose and insulin were increased significantly in non-treated diabetic rats $(p$-value $<0.05)$ that showed the induction of diabetes type 2 in rats. The results showed that in VS-treated diabetic rats compared to the non-treated diabetic group, vanadyl sulfate significantly reduced the glucose and insulin secretion and changed renal inflammatory and oxidative markers, except protein carbonyl so that we couldn't find any significant changes. Our study showed that vanadyl supplementation had positive effects on oxidative stress and inflammation biomarkers in kidney of diabetic rats.
\end{abstract}

Keywords: Vanadyl Sulfate, IL-1, IL-10. Oxidative Stress. Inflammations.

\section{INTRODUCTION}

Hyperglycemia can lead to diabetic problems and their socio-economic burden. Various tissues which have been exposed to high concentration of glucose may lead to changes in cell signaling, increment of oxidizing species or significant reduction in antioxidant capacity. Also, hyperglycemia has critical role in Advanced Glycation End products (AGEs) formation, as well as, secretion of the pro-inflammatory cytokines and cellular death (Volpe et al., 2018). Diabetic Kidney Disease (DKD) is one of the most common complications of

*Correspondence: S.M. Mirhashemi, Metabolic Diseases Research Center, Qazvin University of Medical Sciences, Qazvin, Iran. Tel: +98 28 33337006. E-mail: mirhashemism@gmail.com/Sm.mirhashemi@ qums.ac.ir diabetes, and its severity has increased throughout the world over the last decade due to the growing trend of diabetes, as it is becoming a major cause of death and mortality (Li, Ma, 2017; Liang, Cai, Chen, 2017). It has been documented that trace elements are required for proper function of metabolic pathways (Panchal, Wanyonyi, Brown, 2017; Mirhashemi, Aarabi, 2011). Vanadium, the $3 \mathrm{~d}$ transition metal, has been considered as micro supplements for various biological functions in humans (Petanidis et al., 2013; Pessoa et al., 2015; Levina, Lay, 2017). Main attention to vanadium has focused on anti-diabetic effects but shifted to anti-cancer and anti-parasitic drugs (Levina, Lay, 2017; Kioseoglou et al., 2015). Vanadyl sulfate has been used in humans in the form of insulin-mimetic salt and thus controls both DM type 1 and type 2 . Vanadyl sulfate protects 
from beta cells of Langerhans, and therefore provides insulin production and secretion that can improve blood glucose levels. (Dabros et al., 2004; Ahmadi et al., 2010; El-Shenawy, Refat, Fakihi, 2013) (1-3). Due to the rising occurrence of DKD, multiform study aimed at aborting and remedy is one of the universal priorities for researches. To the best of our facts, this is among the first studies to evaluate vanadyl sulfate on biomarkers of oxidative stress and inflammation in kidney of diabetic rats.

\section{MATERIAL AND METHODS}

All chemicals used in this study were purchased from Sigma-Aldrich Corporation.

\section{Experimental animals}

Healthy male Wistar rats, weighting about 180$210 \mathrm{~g}$, were prepared from Experimental Animal Centre of Kashan University of Medical Sciences, and kept at standard conditions $22-24{ }^{\circ} \mathrm{C}, 40-60 \%$ relative humidity and $12 \mathrm{~h}$ light-dark cycle and fed with standard rat food and water ad libitum. The animals were protected in agreement with the ethics of care and use of experimental animals. The study protocol was permitted by the ethics committee of Kashan University of Medical Sciences (IR.KAUMS.REC.1395.161).

\section{Induction of diabetes type 2 in rats}

A typical diet of high fat (including 400 grams of sheep fat, 200 grams of sucrose, 18 egg yolks and 5 egg whites and 400 grams of rat chow, well combined and homogenized) and high fructose (including 25\% fructose in drinking water) (HFFD: high fat and high fructose diet) was used for 12 weeks in order to generate diabetes type 2 model.

\section{Studied groups design}

30 rats were randomly divided into 3 equal groups as follows. The first group was normal rats that received standard rat chow and water without any HFFD/ VS, and considered as control group. The 2nd group received HFFD without any treatment (non-treated diabetic group) and the 3rd group, received HFFD and $25 \mathrm{mg} / \mathrm{kgBW}$ vanadyl sulfate (diabetic treated with VS25).

\section{Preparation of sera and kidney homogenate}

After 12 weeks, at the end of their treatment periods, the animals were weighed and anesthetized using ether and killed by decapitation. Blood samples were collected; sera were separated from blood cells by centrifugation (Hettich D-78532, Tuttlingen, Germany) at $3000 \mathrm{rpm}$ for $15 \mathrm{~min}$, and stored at $-80 \mathrm{oC}$ until the time of analysis. The kidneys were washed with cold saline $\left(4{ }^{\circ} \mathrm{C}\right)$ and crushed into smaller pieces on ice, homogenized $(10 \% \mathrm{~W} / \mathrm{V})$ with phosphate buffer with 0.1 molar and then centrifuged at $3000 \mathrm{~g}$ for 10 minutes at $4{ }^{\circ} \mathrm{C}$. The supernatant solution was separated from the precipitate and used for measuring the parameters (Mirhashemi et al., 2009).

\section{Glucose and insulin assay}

Fasting blood glucose level, was determined in serum using commercially available kit (Pars Azmun, Tehran, Iran) by Mindray auto analyzer. Insulin level was quantified by ELISA method using kit of DiaMetra Company (Spello, Perugia, Italy).

\section{Renal tissue oxidative biomarkers assay}

Renal Protein Carbonyl (PC) and Malondialdehyde (MDA) were measured by colorimetric method using cayman chemical (USA) and Zellbio GmbH (Germany) commercially available kit with inter- and intraassay CVs of lower than 5\%, respectively. Superoxide Dismutase (SOD) activity was measured using Zellbio (Germany) company kit, too.

\section{Inflammatory biomarkers assay}

Renal tissue IL-1 and IL-10 concentrations were determined using ELISA kit (IBL International Company, Germany) with inter- and intra-assay CVs of 7.5 and $9.1 \%$, respectively.

\section{Statistical analysis}

Data Analysis was performed using the statistical package for social science version 23 (SPSS Inc., Chicago, Illinois, USA). We conducted the Kolmogrov-Smirnov test to evaluate the normality of distribution of variables. Significance of differences was determined by ANOVA and posthoc Tukey's test. Results were presented as the 
Mean \pm SEM and statistically determined significant at $\mathrm{p} \leq 0.05$.

\section{RESULTS}

\section{Effect of vanadyl sulfate on glucose, insulin and HOMA-IR of studied groups}

As it has been shown in Table I, there were statistically significant increase (p-value $<0.001)$ in glucose, insulin and HOMA-IR levels in 2nd group compared to controls $(88.51 \%, 81.6 \%$ and $185.3 \%$ enhancements, respectively). Treatment with vanadyl sulfate resulted in a significant decrease in glucose, insulin and HOMA-IR levels in the 3rd group compared to the 2nd group by $49.21 \%$ (p-value $<0.001$ ), $17.55 \%$ $(\mathrm{p}$-value $=0.012)$ and $31.9 \% \quad(\mathrm{p}$-value $=0.002)$, respectively (Table I).

TABLE I - Effects of HFFD and vanadyl sulfate on fasting blood glucose, Insulin and HOMA-IR in experimental groups

\begin{tabular}{lccc} 
Groups & Glucose $(\mathbf{m g} / \mathbf{d L})$ & Insulin $(\mu \mathbf{I U} / \mathbf{m L})$ & HOMA-IR \\
\hline Control & $110.91 \pm 7.41$ & $1.26 \pm 0.04$ & $0.34 \pm 0.04$ \\
& & & \\
Diabetics & $209.08 \pm 11.60^{*}$ & $1.88 \pm 0.11^{*}$ & $0.97 \pm 0.07^{*}$ \\
VS25 & $171.5 \pm 9.46^{*}$ & $1.55 \pm 0.07^{*}$ & $0.66 \pm 0.05^{*}$
\end{tabular}

All groups were fed with HFFD except controls, for 12 weeks. Treated groups received $25 \mathrm{mg} / \mathrm{kg}$ b.w. of vanadyl sulfate (VS25) in addition to HFFD for the same time Data have been shown as Mean \pm SEM, $\mathrm{n}=10$.

*Indicated significant variations $(\mathrm{p}<0.05)$

\section{Effects of VS-treatment on oxidative stress biomarkers of renal tissue}

According to table II and respect to control group, oxidative biomarkers including MDA and PC were statistically increased significant by $31.51 \%$ and $18.12 \%$ (p-value $<0.001$ ) but SOD activity was decreased by $27.59 \%$ ( $p$-value $<0.001$ ) in the 2 nd group. Vanadyl sulfate reduced MDA and PC in the $3 \mathrm{rd}(5.14 \%$ \& $5.23 \%$, respectively) group compared to the 2 nd group, but these changes were statistically significant only for MDA $(p$-value $=0.029)$ not for PC $(p$-value $=0.28)$. Our results also showed that SOD activity was significantly increased by vanadyl sulfate in VS-treated group by $114.29 \%$ (p-value $<0.001$ ).

TABLE II - Effects of HFFD and vanadyl sulfate on oxidative stress biomarkers in kidney of experimental groups

\begin{tabular}{lccc} 
Groups & SOD $(\mathbf{i U} / \mathbf{m L})$ & MDA $(\boldsymbol{\mu m o l} / \mathbf{L})$ & $\mathbf{P C}(\mathbf{n m o l} / \mathbf{m g})$ \\
\hline Control & $0.29 \pm 0.01$ & $6.82 \pm 0.07$ & $2.35 \pm 0.09$ \\
Diabetics & $0.21 \pm 0.02^{*}$ & $8.94 \pm 0.11^{*}$ & $2.87 \pm 0.06^{*}$ \\
VS25 & $0.45 \pm 0.01^{*}$ & $8.48 \pm 0.15^{*}$ & $2.72 \pm 0.04$ \\
\hline
\end{tabular}

All groups were fed with HFFD except controls, for 12 weeks.

Treated groups received $25 \mathrm{mg} / \mathrm{kg}$ b.w. of vanadyl sulfate (VS25) in addition to HFFD for the same time.

Data have been shown as Mean \pm SEM, $n=10$.

*Indicated significant variations $(\mathrm{p}<0.05)$

\section{Effects of treatment with vanadyl sulfate on renal inflammatory markers}

Compared to control group, high fat and fructose diets led to an increase in IL-1 by $421.95 \%$ (p-value $<0.001$ ) and a decrease in IL-10 by $41.3 \%$ (p-value < $0.001)$ in diabetic group. A significant ( $\mathrm{p}$-value $<0.001$ ) reduction in IL-1 (21.18\%) and increment in IL-10 $(42.23 \%)$ were observed in VS-treated diabetic group compared to diabetics (Table III).

TABLE III - Effects of HFFD and vanadyl sulfate on inflammatory biomarkers in kidney of experimental groups

\begin{tabular}{lcc}
\hline Groups & IL-1 $(\mathrm{pg} / \mathrm{mL})$ & IL-10 $(\mathrm{pg} / \mathrm{mL})$ \\
\hline Control & $1.23 \pm 0.07$ & $3.51 \pm 0.06$ \\
Diabetics & $6.42 \pm 0.08^{*}$ & $2.06 \pm 0.04^{*}$ \\
VS25 & $5.06 \pm 0.07^{*}$ & $2.93 \pm 0.04^{*}$ \\
\hline
\end{tabular}

All groups were fed with HFFD except controls, for 12 weeks. Treated groups received $25 \mathrm{mg} / \mathrm{kg}$ b.w. of vanadyl sulfate (VS25) in addition to HFFD for the same time

Data have been shown as Mean \pm SEM, $n=10$.

*Indicated significant variations $(\mathrm{p}<0.05)$ 


\section{DISCUSSION}

DKD has been considered as one of the most current complication of diabetes and clarified an important factor for death and mortality (Zac-Varghese, Winocour, 2018; Mirhashemi et al., 2016). It has been documented that after developing to renal failure, over $70 \%$ of DKD patients will die within five years (Zarqami et al., 2018), so new treatments for slowing down this progression are an urgent need that should be taken seriously. According to our knowledge, the present study was one of the first studies that evaluated the result of vanadyl sulfate on inflammatory and oxidative stress parameters in kidney of diabetes type 2 models. The first series of results showed that compared to controls, glucose and insulin were increased significantly in non-treated diabetic rats (p-value $<0.001$ ) that showed the induction of diabetes type 2 in rats. Further, the results of vanadyl effects on these two items showed that vanadyl sulfate significantly reduced glucose and insulin secretion. In agreement with our study, Zarghami and colleges showed that vanadyl sulfate supplements may lead to glucose consumption improvement and feed efficiency (Zarqami et al., 2018). In kidney tissue of VS-treated rats, biomarkers of inflammations and oxidative stress were significantly improved, except for the amount of PC that did not change statistically. Oxidative stress and inflammation have been associated with the pathophysiology of diabetic nephropathy (Breyer, Kretzler, 2018; Sun et al., 2013). Studies about the experimental animal models of diabetes strongly implicated oxidant species as a major determinant in the pathophysiology of diabetic kidney disease (Breyer, Kretzler, 2018). In recent years, vanadium compounds have become important for a wide range of diseases and its interaction with cytokine interleukins has been shown (Lin et al., 2018; Sagoo, Gnudi, 2018). Using insulin signaling regulation, Liu and colleagues introduced a new complex of vanadium that resulted in improved performance of diabetic kidney rats. (Tsave et al., 2016). Other authors have also described new findings from various studies about the complex effects of insulin-mimetic vanadium in diabetic rodents (Pelletier et al., 2016; Liu et al., 2014; Jiang et al., 2016). Our study had some limitations. The main limitation of our study was lack of measuring the serum levels of vanadyl sulfate in animals due to financial constraints. Indeed, given the limited financial resource available for these projects, we did not evaluate some of the markers. Therefore, measurement of inflammatory cytokines and biomarkers of oxidative stress after intervention were reasonable in forthcoming studies. It may be concluded that vanadyl sulfate should be a necessary and beneficial molecule for the development of the therapeutic agents for the improvement of oxidative and inflammatory biomarkers in diabetic kidneys. Undoubtedly, further studies are needed to reveal the potential and/or possible toxicity of this compound in long-term use.

\section{ACKNOWLEDGMENT}

We respect the supports of Research Vice Chancellor of Kashan University of Medical Sciences for this project (NO\#95158).

\section{CONFLICT OF INTEREST}

None confirmed.

\section{REFERENCES}

Ahmadi S, Karimian S, Sotoudeh M, Bahadori M, Dehghani G. Pancreatic islet beta cell protective effect of oral vanadyl sulphate in streptozotocin-induced diabetic rats, an ultrastructure study. Pak J Biol Sci. 2010;13(23):1135-40.

Breyer MD, Kretzler M. Novel avenues for drug discovery in diabetic kidney disease. Expert Opin Drug Discov. 2018;13(1):65-74.

Dabros W, Goc A, Turyna B, Kordowiak AM. Influence of vanadyl sulphate [VOSO4] on biochemical activity and morphology of control and streptozotocin-diabetic rat liver Golgi complexes. Pol J Pathol. 2004;55(1):25-32.

El-Shenawy NS, Refat MS, Fakihi FH. Decreasing the diabetic complication by vanadyl (VO) 2+/vitamin B6 complex in alloxan-induced diabetic mice. J Mat Sci Mat Med. 2013;24(4):911-30.

Jiang P, Dong Z, Ma B, Ni Z, Duan H, Li X, Wang B, Ma $\mathrm{X}$, Wei Q, Ji X, Li M. Effect of vanadyl rosiglitazone, a new insulin-mimetic vanadium complexes, on glucose homeostasis of diabetic mice. Appl Biochem Biotechnol. 2016;180(5):841-851.

Kioseoglou E, Petanidis S, Gabriel C, Salifoglou A. The chemistry and biology of vanadium compounds in cancer therapeutics. Coord Chem Rev. 2015;301-302:87-105.

Levina A, Lay PA. Stabilities and biological activities of vanadium drugs: what is the nature of the active species? Chem Asian J. 2017;12(14):1692-1699. 
Evaluating the effects of vanadyl sulfate on biomarkers of oxidative stress and inflammation in renal tissue of rats with diabetes type 2

Li PK, Ma TK. Global impact of nephropathies. Nephrology (Carlton). 2017;22(Suppl 4):9-13.

Liang S, Cai GY, Chen XM. Clinical and pathological factors associated with progression of diabetic nephropathy. Nephrology (Carlton). 2017;22(Suppl 4):14-19.

Lin YC, Chang YH, Yang SY, Wu KD, Chu TS. Update of pathophysiology and management of diabetic kidney disease. J Formos Med Assoc. 2018;117(8):662-675.

Liu Y, Chen DD, Xing YH, Ge N, Zhang Y, Liu J, Zou W. A new oxovanadium complex enhances renal function by improving insulin signaling pathway in diabetic mice. J Diabetes Complications. 2014;28(3):265-72.

Mirhashemi S.M., Moshtaghie, A.A., Ani, M., Aarabi, M.-H. Changes in serum, liver, and brain high and low molecular weight alkaline phosphatase following manganese toxicity in rats. Toxicol Environ Chem. 2009:91(9):349-355.

Mirhashemi SM, Aarabi M-H. To determine the possible roles of two essential trace elements and ascorbic acid concerning amyloidal beta-sheet formation in diabetes mellitus. Scient Res Essays. 2011;6(26):5507-12.

Mirhashemi SM, Rahimi F, Soleimani A, Asemi Z. Effects of omega-3 fatty acid supplementation on inflammatory cytokines and advanced glycation end products in patients with diabetic nephropathy a randomized controlled trial. IJKD 2016;10(4):197-204.

Panchal SK, Wanyonyi S, Brown L. Selenium, vanadium, and chromium as micronutrients to improve metabolic syndrome. Curr Hypertens Rep. 2017;19(3):10.

Pelletier J, Domingues N, Castro MM, Ostenson CG. In vitro effects of $€$ bis(1,2-dimethyl-3-hydroxy-4-pyridinonato) oxidovanadium(IV), or $\mathrm{VO}(\mathrm{dmpp}) 2$, on insulin secretion in pancreatic islets of type 2 diabetic Goto-Kakizaki rats. J Inorg Biochem. 2016:154:29-34.
Pessoa JC, Garribba E, Santos MF, Santos-Silva T. Vanadium and proteins: uptake, transport, structure, activity and function. Coord Chem Rev. 2015;301-302:49-86.

Petanidis S, Kioseoglou E, Hadzopoulou-Cladaras M, Salifoglou A. Novel ternary vanadium-betaine-peroxido species suppresses H-ras and matrix metalloproteinase-2 expression by increasing reactive oxygen species-mediated apoptosis in cancer cells. Cancer letters. 2013;335(2):387-96.

Sagoo MK, Gnudi L. Diabetic nephropathy: Is there a role for oxidative stress? Free Radic Biol Med. 2018:116:50-63.

Sun YM, Su Y, Li J, Wang LF. Recent advances in understanding the biochemical and molecular mechanism of diabetic nephropathy. Biochem Biophys Res Commun. 2013;433(4):359-61.

Tsave O, Petanidis S, Kioseoglou E, Yavropoulou MP, Yovos JG, Anestakis D, Tsepa A, Salifoglou A. Role of Vanadium in Cellular and Molecular Immunology: Association with Immune-Related Inflammation and Pharmacotoxicology Mechanisms. Oxid Med Cell Longev. 2016;2016:4013639.

Volpe CMO, Villar-Delfino PH, Dos Anjos PMF, NogueiraMachado JA. Cellular death, reactive oxygen species (ROS) and diabetic complications. Cell Death Dis. 2018;9(2):119.

Zac-Varghese S, Winocour P. Managing diabetic kidney disease. Br Med Bull. 2018:125(1):55-66.

Zarqami A, Ganjkhanlou M, Zali A, Rezayazdi K, Jolazadeh AR. Effects of vanadium supplementation on performance, some plasma metabolites and glucose metabolism in Mahabadi goat kids. J Anim Physiol Anim Nutr (Berl). 2018:102(2):e972-e977.

Received for publication on $05^{\text {th }}$ August 2018 Accepted for publication on $30^{\text {th }}$ September 2018 